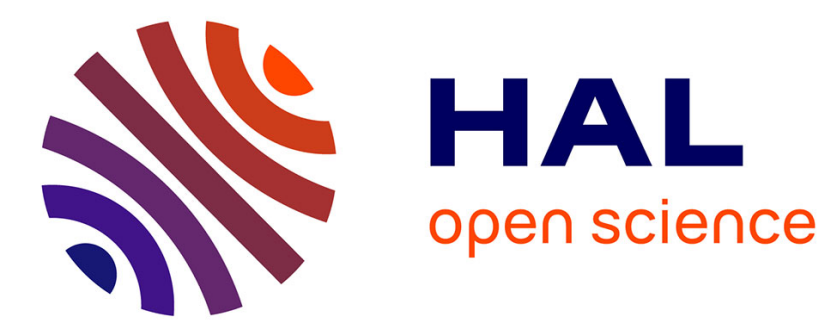

\title{
Magma Ascent and Eruption Triggered by Cratering on the Moon
}

\author{
Chloé Michaut, Virginie Pinel
}

\section{To cite this version:}

Chloé Michaut, Virginie Pinel. Magma Ascent and Eruption Triggered by Cratering on the Moon. Geophysical Research Letters, 2018, 45 (13), pp.6408-6416. 10.1029/2018GL078150 . hal-02327155

\section{HAL Id: hal-02327155 \\ https://univ-lyon1.hal.science/hal-02327155}

Submitted on 3 Sep 2021

HAL is a multi-disciplinary open access archive for the deposit and dissemination of scientific research documents, whether they are published or not. The documents may come from teaching and research institutions in France or abroad, or from public or private research centers.
L'archive ouverte pluridisciplinaire HAL, est destinée au dépôt et à la diffusion de documents scientifiques de niveau recherche, publiés ou non, émanant des établissements d'enseignement et de recherche français ou étrangers, des laboratoires publics ou privés. 


\title{
Geophysical Research Letters
}

\section{RESEARCH LETTER \\ 10.1029/2018GL078150}

\section{Magma Ascent and Eruption Triggered by Cratering on the Moon}

\author{
Chloé Michaut ${ }^{1}$ D and Virginie Pinel $^{2}(\mathbb{D})$ \\ ${ }^{1}$ Ecole Normale Supérieure de Lyon, UCBL, CNRS, Laboratoire de Géologie de Lyon -Terre, Planètes, Environnement, \\ Université de Lyon, Lyon, France, ${ }^{2}$ Université Grenoble Alpes, Université Savoie Mont Blanc, CNRS, IRD, IFSTTAR, ISTerre, \\ Grenoble, France
}

Abstract On the Moon, the low-density crust exerts a strong filter to magma ascent. Many lunar craters are filled with mare, and evidence of pyroclastic activity and shallow magmatism is often located within craters called floor-fractured craters (FFCs). Interpreting quantitative observations on mare-filled craters and FFCs based on mechanical models, we show that a surface unloading caused by an impact crater provides a driving overpressure to the magma stalling at depth. This overpressure counterbalances the melt negative buoyancy, favoring its ascent through the crust. Providing a large unloading and a thin crust, magma can ascend up to the crater floor. FFC characteristics are consistent with a magma denser than the crust by $200-300 \mathrm{~kg} / \mathrm{m}^{3}$ and an elastic lithosphere thickness larger than $70 \mathrm{~km}$. Our study suggests that small impact cratering likely induced magmatism and thereby crustal evolution in the early times of terrestrial planets.

Plain Language Summary On the terrestrial planets showing particularly old surfaces, such as on the Moon, volcanic deposits and evidence of magma intrusions are often found on the floors of relatively small impact craters, too small for the melt to have formed because of the impact itself. Why then is the process of magma ascent and eruption linked to that of cratering? Here we show that the unloading associated to an impact crater decompresses the elastic medium situated beneath. If magma is stalling at depth, it becomes pressurized relatively to its encasing rocks, which allows for its ascent in the crust. Magma might erupt at the surface if the unloading is sufficiently large and the crust relatively thin. Our model well explains observations on evidence of magma eruption versus intrusion at different craters varying by their sizes and by the crustal thickness at their locations on the Moon. We suggest that, in the early times of planets where magmatism and cratering were dominant, magma ascent caused by crater unloading might have modified the initial crustal composition and structure.

\section{Introduction}

The Moon is the only solar system body that presents a well identified primary crust. The lunar highlands, of anorthositic composition, were probably formed very early by flotation of light anorthite minerals on top of a crystallizing magma ocean (Wood et al., 1970) as is attested by their ancient ages (Boyet \& Carlson, 2007; Carlson \& Lugmair, 1988). The lunar crust offers thus the opportunity to study geological processes, characteristics of the early stages of planetary evolution. This primary crust has an average thickness of 34 to $43 \mathrm{~km}$ that varies between less than $1 \mathrm{~km}$ in the basin Moscoviense and up to $60 \mathrm{~km}$ in the farside (Wieczorek et al., 2013). The lunar crust density estimated using gravity data from the NASA's Gravity Recovery and Interior Laboratory mission is on average very low, $\sim 2,550 \mathrm{~kg} / \mathrm{m}^{3}$, because of its anorthositic composition and of its large porosity, a consequence of its intense bombardment (Wieczorek et al., 2013). Crustal density varies laterally by $\pm 250 \mathrm{~kg} / \mathrm{m}^{3}$, with the most mafic exposures found in the south pole Aitken basin. It might vary vertically; however, no systematic deep mafic exposures representative of a lower crust have yet been observed (Besserer et al., 2014; Cahill et al., 2009).

The main driving force for magma ascent is buoyancy, function of the density contrast between the magma and its encasing rocks. The low density of the lunar crust induced a neutral to negative buoyancy for the magma that opposed to its ascent. The basaltic maria indeed preferentially erupted at the lunar surface within large impact basins where part of the crust was removed by impact processes. The solidified grain density of mare basalts varies from $3,270 \mathrm{~kg} / \mathrm{m}^{3}$ for low Ti basalt to $3,450 \mathrm{~kg} / \mathrm{m}^{3}$ for high Ti basalt (Kiefer et al., 2012).

(2018. American Geophysical Union. All Rights Reserved. 
However, basaltic mare magmas at their liquidus have lower densities, estimated between $2,750 \mathrm{~kg} / \mathrm{m}^{3}$ and $3,050 \mathrm{~kg} / \mathrm{m}^{3}$ (Wieczorek et al., 2001).

On planets with no plate tectonics presenting an old, highly craterized surface, volcanic emissions are generally observed within impact craters and basins. On the Moon, pyroclastic deposits, small lava flows, and evidence of magmatism are often located within impact craters (Schultz, 1976; Thomas et al., 2015). The fractured and uplifted floors of some craters (referred to as floor-fractured craters, FFCs) are evidence of crater-centered sill intrusions, (Jozwiak et al., 2012; Thorey \& Michaut, 2014; Thorey et al., 2015; Wichman \& Schultz, 1995). On Mercury as well, traces of pyroclastic flows recently discovered by the MErcury, Surface, Space ENvironment, GEochemistry, and Ranging mission are principally located within impact craters (Kerber et al., 2009; Thomas et al., 2014).

Impact craters unload the crust by redistributing a given mass of material, which decompresses the underlying elastic medium. If melt is being supplied at depth from a mantle source, it becomes pressurized relatively to its encasing rocks. By using mechanical models of the lithospheric stress state coupled with quantitative observations at lunar floor-fractured and mare-filled craters, we present evidence that unloading forces caused by impact craters provided a driving pressure to the magma ponding at the base of the crust, which allowed for its ascent through the crust.

\section{Observations on FFCs}

FFCs were identified and classified into six different classes by Schultz (1976) based on their morphologies and floor appearances and lately reexamined by Jozwiak et al. (2012). Evidence for intrusive activity below FFCs includes radial and concentric fractures on crater floors, uplifted floors, and/or volcanic deposits. Although two different formation mechanisms have been proposed for their formation, there is a general consensus in favor of their magmatic origin (Jozwiak et al., 2012; Thorey \& Michaut, 2014) rather than a viscous relaxation of their floor (Wichman \& Schultz, 1995). About 200 FFCs have been found; they are commonly adjacent to basaltic maria and mascons.

Craters of Classes 2-4 show an uplifted floor, which either appears flat with a U-shaped moat, for FFCs of Class 3 (examples are craters Gassendi and Hutton, Figure 1a,b), or convex up, for FFCs of Classes 2 and 4 (crater Bohnenberger, Figure 1c). Such floor appearances relate to an important intrusion volume. By modeling the dynamics of a crater-centered horizontal intrusion and associated elastic deformation of the crater floor, Thorey and Michaut (2014) have shown that the crater floor appearance can either be flat or convex up depending on the ratio between the flexural wavelength of the layer overlying the intrusion, which mainly depends on its elastic thickness, and the crater radius. The floor appearance is convex for a thick elastic overlying layer or a small crater; it is flat with a U-shaped moat for a thin elastic overlying layer or a large crater (Thorey \& Michaut, 2014). Observations indeed show that craters of Classes 2 and 4 with a convex floor tend to be smaller than craters of Class 3, with a flat floor (Jozwiak et al., 2012; Schultz, 1976). Some FFCs of Class 3 , presenting a flat floor with a U-shaped moat, occur inside mare units and have their floors covered with lavas (see crater Gassendi, for instance, Figure 1a); others present small lava extrusions within the circular moat. For such craters, it is difficult to know which process, from intrusion or extrusion, occurred first and induced the other (see supporting information Table S1).

Mare material has erupted on the floor of craters of Class 6 and along the walls of craters of Class 1. Craters of these classes tend to be very large. They differ from mare-filled craters (such as crater Maksutov, Figure 1d), which also present extrusive mare material on their floors, by the presence of floor fractures indicating some intrusion (supporting information Tables S1 and S2). Their floors are either deep for Class 1 or completely flooded for Class 6 (Jozwiak et al., 2012; Schultz, 1976).

Because craters of Class 5 are old and degraded (Jozwiak et al., 2012), they are not used in this analysis.

Thorey et al. (2015) have extracted the gravitational signatures of crater-centered intrusions by comparing expected signals from intrusion models and observed gravitational anomalies measured by GRAIL at FFCs. Results, for craters in the Highlands, are consistent with the intrusion of mare-type magma in a porous anorthositic crust.

Deformation and uplift at FFCs is usually confined to crater interior: despite shallower floors compared to fresh craters, FFCs generally follow the same rim crest to diameter relationship as the one for fresh craters 


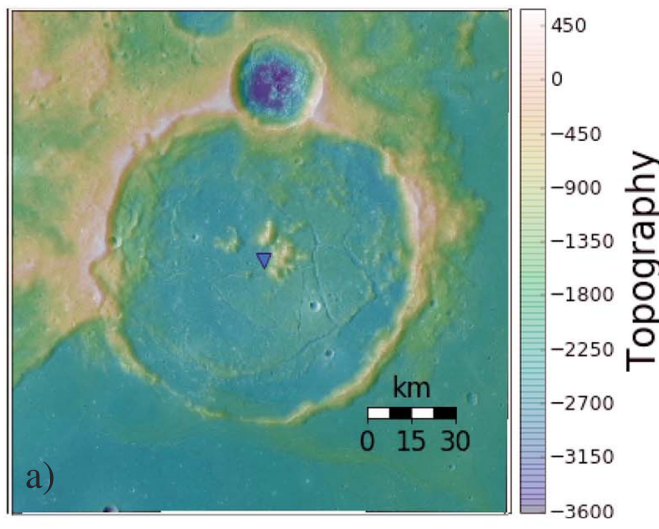

FFC crater Gassendi, Class 3 17.4S 40.0W

floor covered with lava

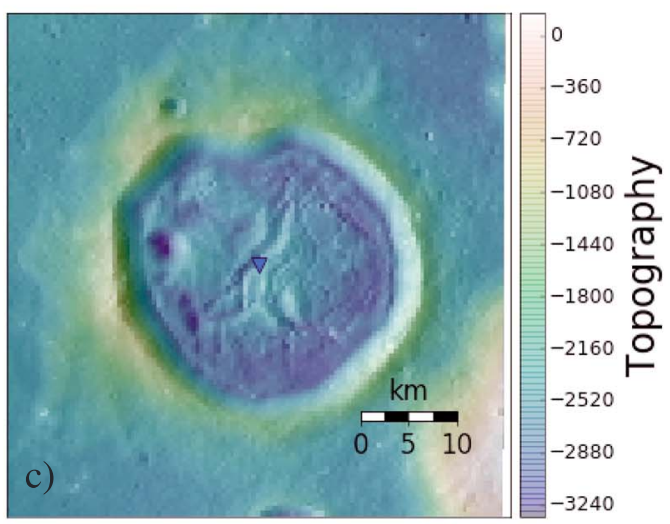

FFC crater Bohnenberger Class 4a 16.2S 40.0E

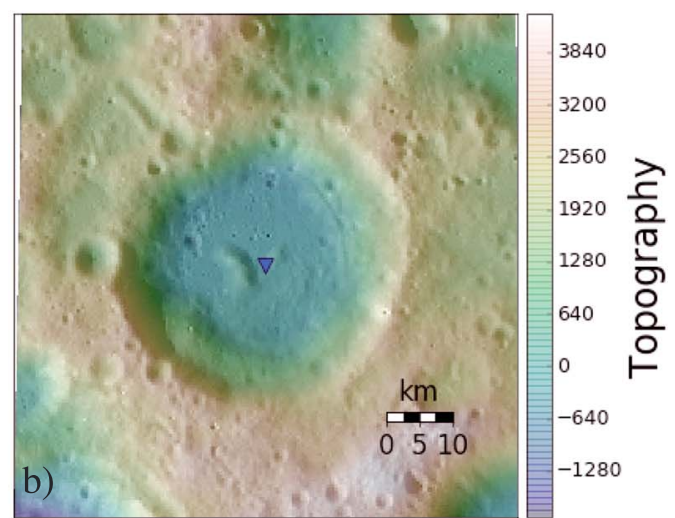

FFC crater Hutton, Class 3 $37.1 \mathrm{~N} 168.6 \mathrm{E}$

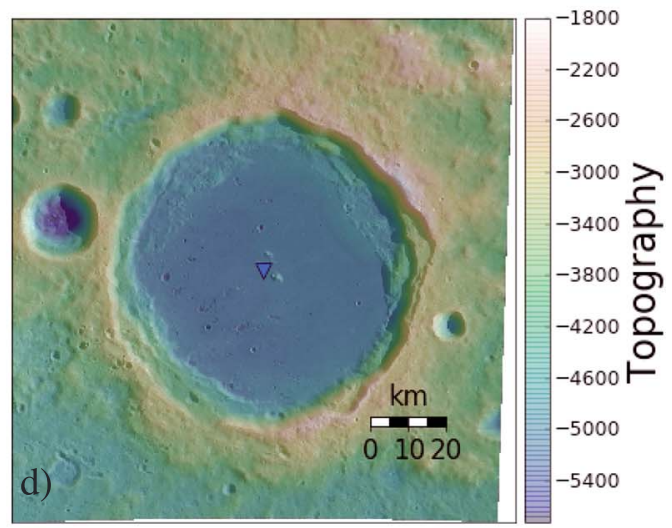

Mare-filled crater Maksutov 40.5S 168.7E

Figure 1. Different examples of mare-filled or floor-fractured craters, see supporting information Table S1 for a summary of the different crater types. The topography is indicated in meter in a color scale; we use the $64 \mathrm{ppd}(472 \mathrm{~m} / \mathrm{pixel})$ gridded data set from the Lunar Orbiter Laser Altimeter (LOLA) mission. FFC = floor-fractured crater.

(Jozwiak et al., 2012; Pike, 1974; Schultz, 1976). This suggests that crater unloading favored and controlled magma ascent below these craters (Pinel \& Jaupart, 2000; Thorey \& Michaut, 2014).

However, it is not clear why the magma is preferentially stored at depth for craters of Classes 2-4. Previous authors have proposed that the presence of a solidified impact melt sheet (Schultz, 1976) or of a strongly brecciated zone (Jozwiak et al., 2012) would constitute a rigidity barrier, for the melt sheet, or a low-density barrier, for the brecciated layer, below which the magma will tend to spread. However, the impact melt sheet should be negligible for the smaller craters of Class 4, which are those associated to the deepest intrusions (Thorey \& Michaut, 2014). Furthermore, the brecciated zone should extend to greater depth and be more porous for larger craters, implying a larger density barrier. Observations show on the contrary that craters of Classes 1 and 6, where magma erupted on the crater floor, tend to be larger than craters with an uplifted floor of Classes 2-4 (Jozwiak et al., 2012; Schultz, 1976).

Craters that present evidence of eruption on their floors are generally large, suggesting that crater size also exerted a strong control on the ability of magma to reach the surface. We present below new evidence that cratering provided an additional pressure to the magma by reducing the pressure of its encasing rocks, driving its ascent in the low-density lunar crust. 


\section{Perturbations in the Pressure Field of an Elastic Lithosphere by Crater Unloading}

Impact events create pressure reductions that induce perturbations to the lithospheric stress state. We consider a surface unloading $\sigma_{z z}^{C}(z=0)$ for $0 \leq r \leq R$ caused by a bowl-shape axisymmetric crater of radius $R$ :

$$
\sigma_{z z}^{c}(r, z=0)=-\rho_{c} g h_{0}\left(1-\frac{r^{2}}{R^{2}}\right)
$$

where $h_{0}$ is the maximum crater depth, $\rho_{c}$ is the crustal density, $g$ is the gravity, and $r$ is the radial coordinate. This expression is negative as tensile stresses are considered negative by convention. For simplicity we do not model central peaks, which have a second-order effect on intrusion emplacement (Thorey \& Michaut, 2014). Using different shapes accounting for a larger flat floor characteristic of large complex craters does not significantly change our results (see supporting information Text S1 and Figure S2).

Crater depth increases with crater radius following a power law relationship (Pike, 1974). As we mostly look at craters larger than $20 \mathrm{~km}$, we use the relationship for complex lunar craters in the Highlands determined by Thorey et al. (2015):

$$
h_{0}=540 \times\left(2 \times R \times 10^{-3}\right)^{0.44}
$$

with $R$ and $h_{0}$ in meters. We calculate the stress field $\sigma^{C, T_{e}}$ and pressure $P^{C, T_{e}}$ perturbations caused by this unloading in an elastic plate of finite thickness $T_{e}$ as well as in a semi-infinite medium solving the equations for linear elasticity with the finite element method (COMSOL software, see supporting information Text S1 for more details). We verified that results for large plate thicknesses compared to crater radius tend to the analytical results for a semi-infinite medium (Sneddon, 1951). In particular, the vertical pressure profile $P^{C, \infty}$ in a semi-infinite medium of Poisson's ratio $v$ at depth $z$ and at the axis of a bowl-shape unloading is given by (see supporting information Text S1):

$$
P^{C, \infty}(z)=-\frac{4}{3} \frac{\rho_{c} g h_{0}(1+v)}{R^{2}}\left(\frac{R^{2}}{2}-z \sqrt{R^{2}+z^{2}}+z^{2}\right)
$$

At depth, the decompression $P^{C, T} T_{e}$ scales with the surface unloading (1) and is larger for a larger crater: it decreases over a characteristic depth similar to the crater radius, as long as the plate is thick compared to crater radius, that is, for $T_{e} \geq \frac{3}{2} R$ (Figure 2 ).

In the case of a semi-finite medium, the decompression $P^{C, \infty}$ tends to zero at infinity. For a finite plate thickness, upward bending caused by crater unloading induces a compressive stress state at the plate bottom $\left(P^{C, T_{e}}(z=T e)>0\right)$. The thicker the plate compared to crater radius, the deeper the zone in tension is: it extends over about two thirds of the plate for $T_{e}=1.5 R$ to $3 R$ (Figure 2).

\section{Impact-Induced Eruption Conditions}

If melt is being supplied and ponds at depth in the elastic lithosphere below the crater unloading, it becomes pressurized relatively to its encasing elastic rocks that have seen their pressure decreases by unloading. The maximum melt overpressure at depth $z$ is then equal and opposite to the decompression caused by unloading at this depth: $\Delta P^{m}(z)=-P^{C}(z)$. If the melt is connected to a deeper source or reservoir, its overpressure can easily reach this maximum. If not, part of the environmental decompression could also be transmitted to the melt depending on reservoir geometry and magma compressibility (Pinel \& Albino, 2013). In the latter case, magma overpressure will be larger for vertically elongated magma reservoirs, as expected when a rising dike overshoots the neutral buoyancy level, filled with compressible magma, $\Delta P^{m}(z)$ being then an upper bound.

We assume that magma is positively buoyant in the mantle but negatively buoyant in the crust, which causes melt to pond close to the mantle-crust interface of depth $D_{c}$ in the lunar lithosphere (Head \& Wilson, 1992; Wilson \& Head, 2017). We examine in which cases the pressure reduction caused by a crater within the crust can induce magma ascent up to the crater floor.

If the elastic lithosphere is too thin compared to crust thickness $\left(D_{c}\right.$ is deeper than the zero-pressure limit indicated in Figure 2), $P^{C}\left(D_{c}\right)>0 \Rightarrow \Delta P^{m}\left(D_{c}\right)<0$, and magma ascent is not allowed. If, on the contrary, $T_{e}$ is large enough compared to $D_{c}, P^{C}\left(D_{c}\right)<0$, which provides a positive driving pressure for magma ascent through the crust. The zero-pressure limit is located at a depth of $\sim 2 / 3 \times T_{e}$ (Figures 2 and 3 ). Hence, for a crust 


\section{Cylindrical state of stress}

$-1 \quad-0.8-0.6-0.4-0.2 \quad 0 \quad 0.2$

$\mathrm{P} /\left(\rho_{\mathrm{c}} g \mathrm{~g}_{\mathrm{o}}\right)$
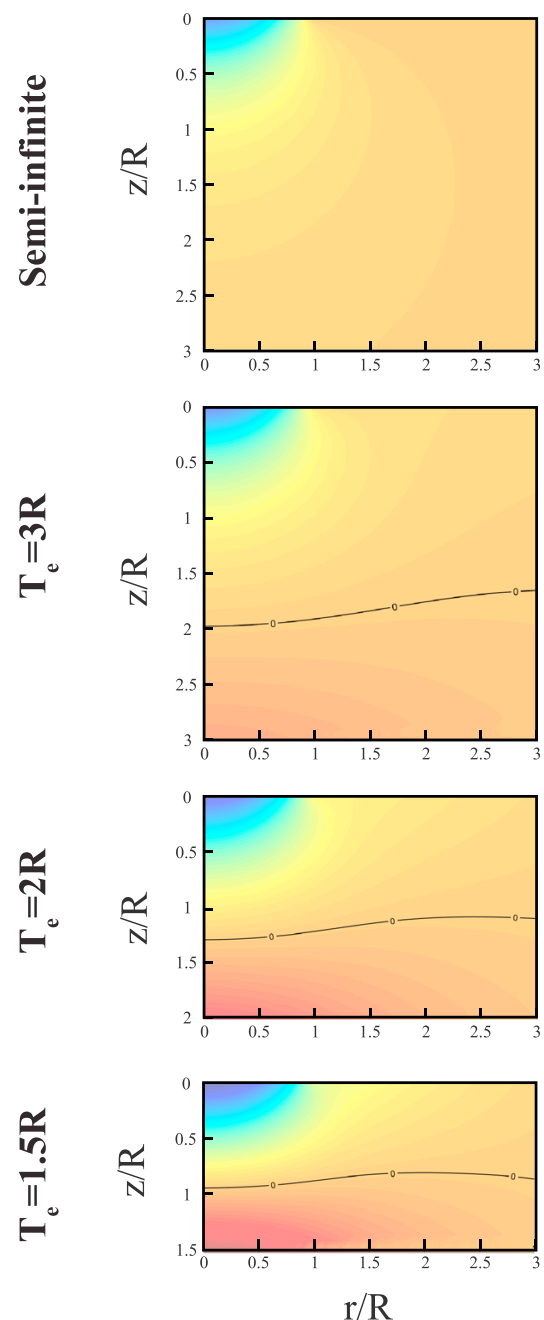

Stress at the axis (normalised by $\rho_{\mathrm{c}} \mathrm{gh}$ )
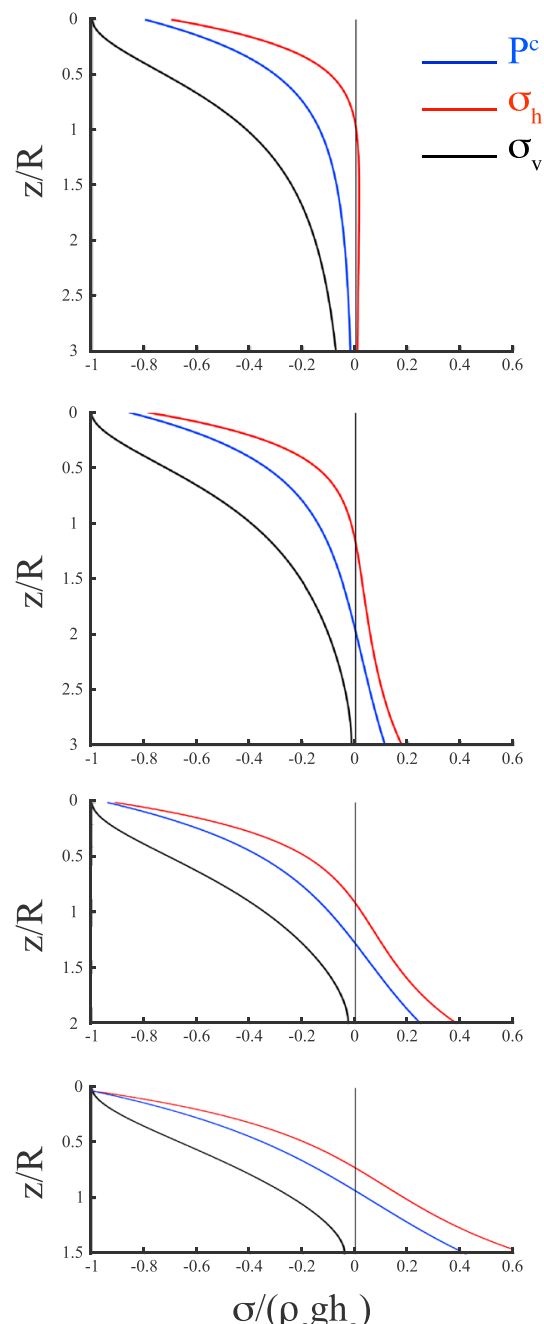

Figure 2. (left column) Pressure perturbation $P^{C}$ (color scale, tension is negative) at depth below a bowl-shape pressure perturbation of radius $R$ and maximum depth $h_{0}$ for a semi-infinite elastic medium (top row) and three different elastic plate thicknesses (from top to bottom): $T_{e}=3 R, 2 R$, and $\frac{3}{2} R$. Black solid line: limit of zero-pressure perturbation. (right column) Horizontal stress $\sigma_{h}=\sigma_{r r}=\sigma_{\theta \theta}$ (red line), vertical stress $\sigma_{v}=\sigma_{z z}$ (black line), and pressure $P^{C}$ (blue line) at the unloading axis, for the same four cases. Pressure is normalized by $\rho_{c} g h_{0}$; depth is normalized by crater radius $R$. Results are for Poisson's ratio $v=0.2$, Young's modulus $E=10 \mathrm{GPa}, g=1.62 \mathrm{~m} / \mathrm{s}^{2}$, lithospheric mantle density $\rho_{L}=3,400 \mathrm{~kg} / \mathrm{m}^{3}$.

thickness between 30 and $50 \mathrm{~km}$, the elastic lithosphere thickness must be larger than 45 to $75 \mathrm{~km}$ to allow for magma ascent below craters (Figures 3 and S3).

For given crater radius $R$ and density difference between the crust and magma $\Delta \rho=\rho_{c}-\rho_{m}<0$, where $\rho_{m}$ is magma density, we invert for the maximum crustal height $D_{c}$ through which the magma can ascend with the overpressure provided by crustal unloading. We account for both the overpressure provided initially to the magma at its location depth and the overpressure acquired during its ascent toward the surface, as the pressure reduction due to unloading increases toward the surface. A magma, initially at a depth $D$, can start its ascent and reach a shallower crustal depth $z$ if $-P^{C}(D)>0$; it can ascend further up as long as the sum of its initial overpressure $-P^{C}(D)$ and of the additional overpressure at its new depth $-P^{C}(z)+P^{C}(D)$ is larger than 

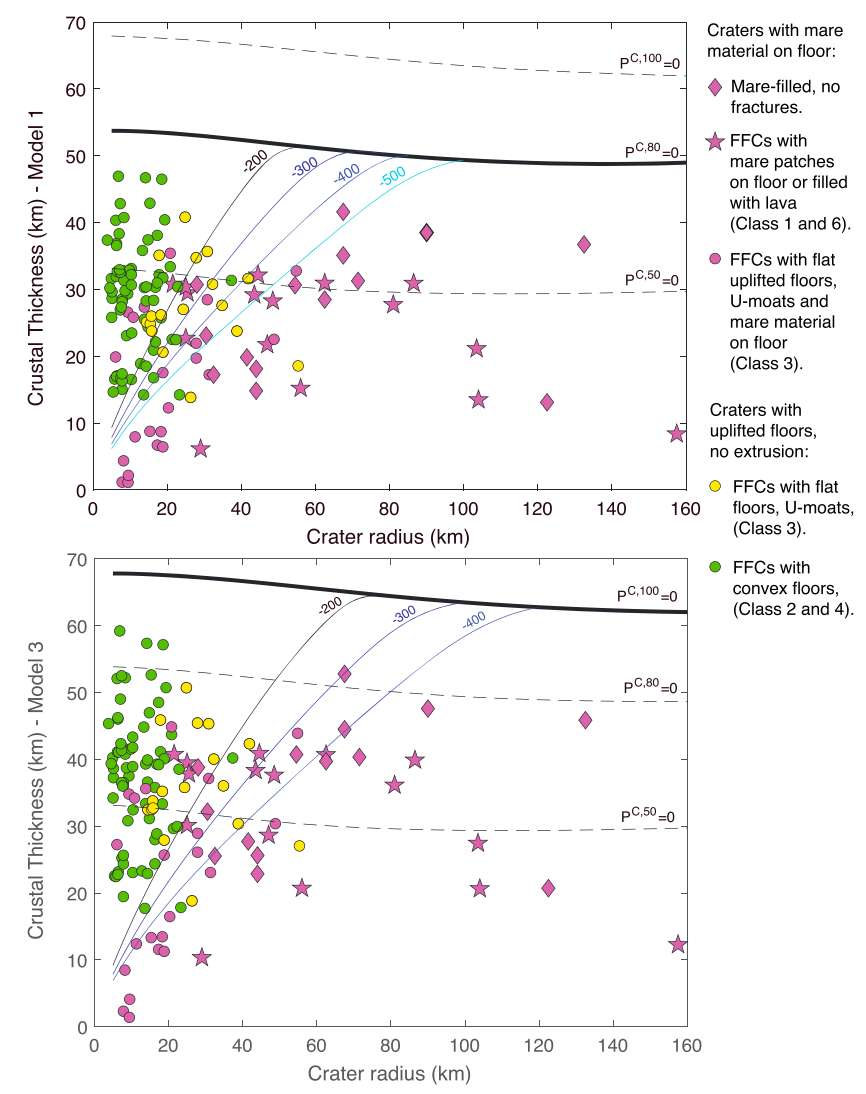

Figure 3. Thin solid lines: maximum height that a negatively buoyant magma can penetrate with an overpressure caused by crater unloading in an elastic plate of thickness $T_{e}=80 \mathrm{~km}$ (top) and $100 \mathrm{~km}$ (bottom) as a function of crater radius, for different values of the product $\Delta \rho g$ indicated on the graph in $\mathrm{Pa} / \mathrm{m}$. The bold and dashed lines indicate the limit of zero-pressure perturbation due to crater unloading for different $T e$ values $(50,80$, and $100 \mathrm{~km})$ indicated on the graph. Crustal thickness at the location of FFCs and mare-filled craters are plotted as a function of crater radius for different crater types (see legend on the right). Top panel is for crustal Model 1 (Wieczorek et al., 2013), for which the average crustal thickness is $34 \mathrm{~km}$, the thickness at Apollo 12/14 site is $29.9 \mathrm{~km}$, the minimum thickness is $0.6 \mathrm{~km}$, and the mantle density is $3,220 \mathrm{~kg} / \mathrm{m}^{3}$. Bottom panel is for crustal Model 3, for which the average thickness is $43 \mathrm{~km}$, the thickness at Apollo 12/14 site is $38.1 \mathrm{~km}$, the minimum thickness is $1.0 \mathrm{~km}$, and the mantle density is $3,150 \mathrm{~kg} / \mathrm{m}^{3}$. Both models consider a crustal porosity of $12 \%$. Results are for $v=0.2, E=10 \mathrm{GPa}, g=1.62 \mathrm{~m} / \mathrm{s}^{2}$, and $\rho_{L}=3220 \mathrm{~kg} / \mathrm{m}^{3}$. FFCs $=$ floor-fractured craters. its negative buoyancy over the height $(D-z)$ it has reached. The maximum crustal height $D_{c}$ should thus verify

$$
\begin{aligned}
& \forall D \leq D_{c} \quad \forall z \in[0, D] \quad-P^{C}(D)+\Delta \rho g(D-z)-P^{C}(z)+P^{C}(D)>0 \\
& \Leftrightarrow+\Delta \rho g(D-z)-P^{C}(z)>0 \\
& \text { For } \quad D>D_{c} \quad \exists z \in[0, D] \quad+\Delta \rho g(D-z)-P^{C}(z)<0
\end{aligned}
$$

where $P^{C}(z)$ is calculated from (3) for a semi-infinite layer or using COMSOL (see previous section) for a finite plate thickness.

The larger the crater radius, the larger the overpressure provided to the magma by unloading, and the larger the crustal height the magma can penetrate (Figure 3). This height increases as the negative difference in density between the crust and magma decreases (Figure 3).

As expected, results for a semi-infinite medium and a finite plate thickness converge for small crater radii and diverge for crater diameters larger than $\sim T_{e}$ (supporting information Figure $\mathrm{S} 1$ ). The larger the elastic lithosphere thickness, the larger the depth of the zero-pressure limit below which no ascent is allowed (Figures 3 and S3).

We use crustal thickness models obtained from GRAIL's gravity data (Wieczorek et al., 2013) to determine the crustal thickness at the location of each floor-fractured or mare-filled crater (Jozwiak et al., 2012) as a function of crater radius and crater type (see Table S1 summarizing crater types and Figure 3). Models 1 and 3 mainly differ by their average and Apollo 12/14 crustal thickness (see caption of Figure 3 and Wieczorek et al. (2013) for more details).

Almost all craters showing eruption of basaltic material on their floor, that is, mare-filled craters, FFCs of Classes 1 and 6, and some FFCs of Class 3, are relatively large craters that induce a rather large pressure reduction in the underlying crust (pink symbols in Figure 3). They tend to plot below the theoretical solid curves for a relevant elastic thickness value $\left(T_{e}>80 \mathrm{~km}\right.$ for Model 1 and $T_{e}>100 \mathrm{~km}$ for Model 3). This demonstrates that the additional overpressure provided by crater unloading was large enough to allow for magma ascent from the base of the crust up to the surface at these craters.

On the contrary, FFCs showing uplifted floors, that is, evidence of intrusions, and no significant extrusion of lavas (Jozwiak et al., 2012; Schultz, 1976; Thorey \& Michaut, 2014), are in general small craters on a relatively thick crust (yellow and green circles in Figure 3); they tend to plot above theoretical curves. This suggests that the overpressure provided by crater unloading was not large enough to cause magma eruption; magma ascended through the crust but ponded at shallow depths. Furthermore, FFCs of Classes 2 and 4, showing a convex floor characteristic of a smaller crater or deeper intrusion, that is, a smaller overpressure (Thorey \& Michaut, 2014), plot on the far left of these curves, while FFCs of Class 3, with a flat uplifted floor characteristic of a larger crater or shallower intrusion, that is, a larger overpressure, have an intermediate position. They stand between craters with a convex floor (green circle) and craters showing eruptions on their floors (pink symbols), close to the theoretical curves (Figure 3).

Crustal thickness estimates from GRAIL's data vary by $\sim 10 \mathrm{~km}$ depending on parameter values, and crustal density also varies laterally (Wieczorek et al., 2013), which might explain why some FFCs of Classes 1 and 6 plot above and some FFCs of Class 3 plot below theoretical curves. Furthermore, for craters of Class 3 presenting mare deposits on their floor (pink circles), it is difficult to evaluate if extrusion occurred following intrusion, because of associated fracturing and magma differentiation, or if eruption occurred first. 


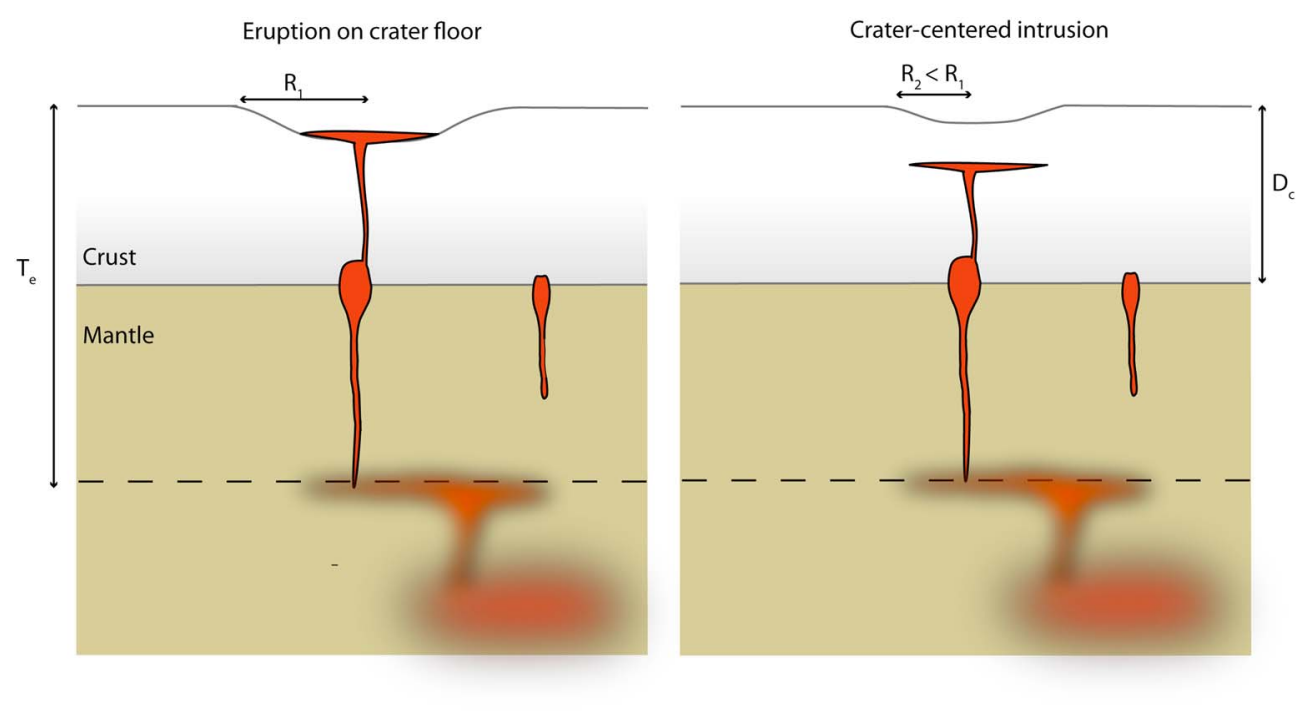

Figure 4. Schematics summarizing the process of magma ascent by crater unloading and eruption on crater floor or crater-centered intrusion.

Moreover, no mare-filled or FFCs plot above the zero-pressure perturbation limit, where the stress state below an unloading is compressive leading to magma underpressurization, for a relevant elastic thickness value ( $T_{e} \gtrsim 80 \mathrm{~km}$ for Model 1 and $T_{e} \gtrsim 100 \mathrm{~km}$ for Model 3, bold lines in Figure 3).

FFC and mare-filled crater characteristics are thus consistent with magma ascent being driven by crater unloading, which would also explain why magma eruptions and floor deformations are generally constrained to crater interior (Jozwiak et al., 2012; Schultz, 1976; Thorey \& Michaut, 2014).

\section{Discussion and Conclusion}

We have demonstrated that crater unloading in a lunar elastic lithosphere thicker than $\sim 75-80 \mathrm{~km}$ provided an additional overpressure to the magma supplied from the mantle that allowed for magma ascent through the crust. Eruption at the surface was possible if magma was stalling below a relatively thin crust and a large crater and was still connected to a deeper source or forming an independent vertically elongated storage zone (Figure 4). For smaller craters and/or thicker crust, the overpressure was smaller and magma formed an intrusion (Figure 4).

Elastic lithosphere thickness values $\gtrsim 75-80 \mathrm{~km}$ are consistent with magma intruding below FFCs after the main stage of mare volcanism. Indeed, smaller values of $\sim 25$ to $75 \mathrm{~km}$, characteristics of hotter lithosphere and earlier times, are obtained by comparing tectonic observations and models of basin loading by lava infilling (McGovern \& Litherland, 2011; Solomon \& Head, 1979). An elastic thickness of $T_{e}=50 \mathrm{~km}$ is however inconsistent with FFC data since many FFCs would plot above the zero-pressure limit (Figures 3 and S3). Spatial differences in elastic thickness are also likely on the Moon, since heat producing elements appear concentrated in the Oceanus Procellarum Kreep Terrane, which might explain part of this discrepancy (Thiriet et al., 2018), though many FFCs are located close to this province.

This work suggests that crater-centered magmatism is triggered by and occurs following cratering. Crater counts at the crater Tsiolkovsky indeed indicates that mare lavas were erupted soon after crater formation (Mouginis-Mark \& Boyce, 2017). However, there is a significant time span over which crater unloading may induce magma ascent that should scale with the timescale of viscoelastic relaxation of the elastic lithosphere and crust. This one depends on the lithosphere thermal state but is particularly large for the Moon, larger than for the Earth, because of its small radius (Zhang \& Zuber, 2000). On the Earth, this timescale is estimated around 1 to 10 Myrs based on lithospheric flexural studies (Beaumont, 1981; Nunn \& Sleep, 1984) and interpretation of vents distribution from models of dike paths (Maccaferri et al., 2017). On the Moon, complex craters in the Highlands generally show negative Bouguer gravity anomalies independently of their age and intermediate-sized craters seem only partially compensated (Reindler \& Arkani-Hamed, 2001; Soderblom et al., 2015; Thorey et al., 2015), suggesting that the crust can support the stress caused 
Acknowledgments

We thank Laurent Montési for his thorough review of the manuscript as well as an anonymous reviewer. This work was supported by PNP/INSU/CNES. We use the lunar floor-fractured crater data set produced by Jozwiak et al. (2012) and stored in run: http://www.planetary.brown.edu/ html_pages/ffc.html. by unloading over a timescale longer than $10^{8}$ years. The mechanism we describe can account for the observed compensation of small- to intermediate-sized craters (Reindler \& Arkani-Hamed, 2001). Many complex craters show a positive anomaly; the mean Bouguer anomaly of nonmodified craters is positive for crater diameters $\lesssim 40 \mathrm{~km}$. It has been suggested that these positive values result from impact-induced compaction in a highly porous target (Soderblom et al., 2015). Our study suggests that they could also result from deep crater-centered intrusions that do not lead to significant floor uplift or deformation (Zhang et al., 2018).

Our results point to a density difference between the crust and magma of $200-300 \mathrm{~kg} / \mathrm{m}^{3}$. Such values are consistent with density estimates of basaltic melts from their chemical composition, that are larger than $2,750 \mathrm{~kg} / \mathrm{m}^{3}$ (Wieczorek et al., 2001). At low pressures $<40 \mathrm{MPa}$, that is, depths $<8 \mathrm{~km}$, magma ascent might have been helped punctually by CO exsolution (Nicholis \& Rutherford, 2009; Sato, 1979), which could explain some of the discrepancies between theory and data (Figure 3).

Our study also suggests that the magma erupting on crater floors was tapped from deep in the crust (Figures 3 and 4). Where produced, the magma ascended in the mantle by buoyant flow and probably ponded close to the mantle-crust interface (Head \& Wilson, 1992; Wilson \& Head, 2017). Buoyancy excess in the mantle might have helped magma rising within the crust (Wilson \& Head, 2017). Data show that intrusions formed below craters on a crust as thin as $13 \mathrm{~km}$ (Figure 3), suggesting that rising dikes do not ascend more than $\sim 10 \mathrm{~km}$ within the crust. Accounting for crustal magma ascent by buoyancy excess in the mantle would thus amount to decrease by $\sim 10 \mathrm{~km}$ the thickness of the crustal column at the location of FFCs and mare-filled craters (Figure 3) and thereby to decrease the minimum elastic thickness $T_{e}$ by $\sim 6-7 \mathrm{~km}$ and to raise the estimated density difference between the crust and magma by $\lesssim 200 \mathrm{~kg} / \mathrm{m}^{3}$.

The Moon example has already shown that secondary crust formation was triggered by large impacts forming basins. Here we show that crustal magmatism, and associated crustal evolution, was also made possible by small impact processes in the early times of the Moon and probably of the other planets as well.

\section{References}

Beaumont, C. (1981). Foreland basins,. Geophysical Journal of the Royal Astronomical Society, 65, 291 - 329.

Besserer, J. F., Nimmo, F., Wieczorek, M. A., Weber, R. C., Kiefer, W. S., McGovern, P. J., et al. (2014). GRAIL gravity constraints on the vertical and lateral density structure of the lunar crust. Geophysical Research Letters, 41, 5771 - 5777. https://doi.org/10.1002/2014GL060240

Boyet, M., \& Carlson, R. W. (2007). A highly depleted Moon or a non-magma ocean origin for the lunar crust? Earth and Planetary Science Letters, 262, 505-516.

Cahill, J. T. S., Lucey, P. G., \& Wieczorek, M. A. (2009). Compositional variations of the lunar crust: Results from radiative transfer modeling of central peak spectra. Journal of Geophysical Research, 114, E09001. https://doi.org/10.1029/2008JE003282

Carlson, R. W., \& Lugmair, G. W. (1988). The age of ferroan anorthosite 60,025: Oldest crust on a young Moon. Earth and Planetary Science Letters, 90, 119-130.

Head, J. W., \& Wilson, L. (1992). Lunar mare volcanism: Stratigraphy, eruption conditions, and the evolution of secondary crusts. Geochimica et Cosmochimica Acta, 56, 2155-2175.

Jozwiak, L. M., Head, J. W., Zuber, M. T., Smith, D. E., \& Neumann, G. A. (2012). Lunar floor-fractured craters: Classification, distribution, origin and implications for magmatism and shallow crustal structure. Journal of Geophysical Research, 117, E11005. https://doi.org/10.1029/2012JE004134

Kerber, L., Head, J. W., Solomon, S. C., Murchie, S. L., Blewett, D. T., \& Wilson, L. (2009). Explosive volcanic eruptions on Mercury: Eruption conditions, magma volatile content, and implications for interior volatile abundances. Earth and Planetary Science Letters, 285, 263-271. https://doi.org/10.1016/j.epsl.2009.04.037

Kiefer, W. S., Macke, R. J., Britt, D. T., Irving, A. J., \& Consolmagno, G. J. (2012). The density and porosity of lunar rocks. Geophysical Research Letters, 39, L07201. https://doi.org/10.1029/2012GL051319

Maccaferri, F., Richeter, N., \& Walter, T. R. (2017). The effect of giant lateral collapses on magma pathways and the location of volcanism. Nature Communications, 8, 1097. https://doi.org/10.1038/s41467-017-01256-2

McGovern, P. J., \& Litherland, M. M. (2011). Lithospheric stress and basaltic magma ascent on the Moon, with implications for large volcanic provinces and edifices. Lunar and Planet Science XLII, Abstract \#2587.

Mouginis-Mark, P. J., \& Boyce, J. M. (2017). Mare infilling of Tsiolkovsky Crater, the Moon. Lunar and Planetary Science Conference XLVIII Abstract \#1206.

Nicholis, M. G., \& Rutherford, M. J. (2009). Graphite oxidation in the Apollo 17 orange glass magma: Implications for the generation of a lunar volcanic gas phase.Geochimica et Cosmochimica Acta, 73, 5905-5917.

Nunn, J. A., \& Sleep, N. H. (1984). Thermal contraction and flexure of intracratonal basins: A three dimensional study of the Michigan basin. Geophysical Journal of the Royal Astronomical Society, 76, 587-635.

Pike, R. J. (1974). Formation of complex impact craters: Evidence from Mars and other planets. Icarus, 43, 1-19.

Pinel, V., \& Albino, F. (2013). Consequences of volcano sector collapse on magmatic storage zones: Insights from numerical modeling Journal of Volcanology and Geothermal Research, 252, 29-37. https://doi.org/10.1016/j.jvolgeores.2012.11.009

Pinel, V., \& Jaupart, C. (2000). The effect of edifice load on magma ascent beneath a volcano. Philosophical Transactions of the Royal Society of London, 358, 1515-1532.

Reindler, L., \& Arkani-Hamed, J. (2001). The compensation state of intermediate size lunar craters. Icarus, 153, 71-88.

Sato, M. (1979). The driving mechanism of lunar pyroclastic eruptions inferred from the oxygen fugacity behavior of Apollo 17 orange glass. In Proceedings of the Lunar Science Conference 10th (pp. 311-325). Houston, TX.

Schultz, P. H. (1976). Floor-fractured lunar craters. Moon, 15, 241-273. 
Sneddon, I. N. (1951). Fourier transforms. New York: Dover Publications.

Soderblom, J. M., Evans, A. J., Johnson, B. C., Melosh, H. J., Miljkovic, K., Phillips, R. J., et al. (2015). The fractured Moon: Production and saturation of porosity in the lunar highlands from impact cratering. Geophysical Research Letters, 42, 6939-6944. https://doi.org/10.1002/2015GL065022

Solomon, S. C., \& Head, J. W. (1979). Vertical movement in mare basins: Relation to mare emplacement, basin tectonics, and lunar thermal history. Journal of Geophysical Research, 84, 1667-1682.

Thiriet, M., Michaut, C., Breuer, D., \& Plesa, A. C. (2018). Hemispheric dichotomy in lithosphere thickness on mars caused by differences in crustal structure and composition. Journal of Geophysical Research: Planets, 123, 823-848. https://doi.org/10.1002/2017JE005431

Thomas, R. J., Rothery, D. A., Conway, S. J., \& Anand, M. (2014). Mechanisms of explosive volcanism on Mercury: Implications from its global distribution and morphology. Journal of Geophysical Research, 119, 2239-2254. https://doi.org/10.1002/2014JE004692

Thomas, R. J., Rothery, D. A., Conway, S. J., \& Anand, M. (2015). Explosive volcanism in complex impact craters on Mercury and the Moon: Influence of tectonic regime on depth of magmatic intrusion. Earth and Planetary Science Letters, 431, $164-172$. https://doi.org/10.1016/j.epsl.2015.09.029

Thorey, C., \& Michaut, C. (2014). A model for the dynamics of crater-centered intrusions: Application to lunar floor-fractured craters. Journal of Geophysical Research: Planets, 119, 286-312. https://doi.org/10.1002/2013JE004467

Thorey, C., Michaut, C., \& Wieczorek, M. (2015). Gravitational signatures of lunar floor-fractured craters. Earth and Planetary Science Letters, 424, 269-279. https://doi.org/10.1016/j.epsl.2015.04.021

Wichman, R. W., \& Schultz, P. H. (1995). Floor-fractured craters in mare smythii and west of Oceanus Procellarum implications of crater modification by viscous relaxation and igneous intrusion models. Journal of Geophysical Research, 100, 21,209-21,218.

Wieczorek, M. A., Neumann, G. A., Nimmo, F., Kiefer, W. S., Taylor, G. J., Melosh, H. J., et al. (2013). The crust of the Moon as seen by GRAIL. Science, 339, 671-675. https://doi.org/10.1126/science.1231530

Wieczorek, M. A., Zuber, M. T., \& Phillips, R. J. (2001). The role of magma buoyancy on the eruption of lunar basalts. Earth and Planetary Science Letters, 185, 71-83.

Wilson, L., \& Head, J. W. (2017). Generation, ascent and eruption of magma on the Moon: New insights into source depths, magma supply, intrusions and effusive/explosive eruptions (Part 1: Theory). Icarus, 283, 146-175.

Wood, J. A., Dickey, J. S., Marvin, U. B., \& Powell, B. N. (1970). Lunar anorthosites and a geophysical model of the Moon. In Proceedings of the Apollo 11 Lunar Science Conference (pp. 1965-1988). Houston, TX.

Zhang, F., Zhu, M.-H., Bugiolacchi, R., Huang, Q., Osinski, G. R., Xiao, L., \& Zou, Y. L. (2018). Diversity of basaltic lunar volcanism associated with buried impact structures: Implications for intrusive and extrusive events. Icarus, 307, 216-234. https://doi.org/10.1016/j.icarus.2017.10.039

Zhang, S., \& Zuber, M. T. (2000). Long-wavelength topographic relaxation for self-gravitating planets and implications for the time-dependent compensation of surface topography. Journal of Geophysical Research, 105, 4153-4164. 